\title{
Variations in Linear and Nonlinear Postural Measurements under Achilles Tendon Vibration and Unstable Support-Surface Conditions
}

\author{
Matthew J. E. Turnock, Charles S. Layne \\ International Space University, Department of Health and Human Performance, Center for Neuromotor and Biomechanics \\ Research, University of Houston, Texas.
}

\begin{abstract}
Reduced support-surface stability has been shown to attenuate the effect of Achilles tendon vibration on backward body displacement. In the present study, 20 participants performed a quiet, upright standing task on a stable and sway-referenced support, with and without vibration. The authors calculated equilibrium scores (ES), approximate entropy (ApEn), and mean and peak power spectral density frequencies of center-of-pressure variations. It was found that ES values decreased with the addition of vibration and in the sway-referenced support condition. ApEn values decreased with the addition of vibration but only with a stable support. Conversely, mean and peak frequencies increased with the addition of vibration, independent of support stability. These results suggest that the role of ankle proprioceptive input changes depending on support-surface characteristics and demonstrate the value of using both linear and nonlinear measures of postural sway.
\end{abstract}

Keywords: nonlinear measures, posture, proprioception, surface stability, vibration

$\mathbf{P}$ ostural control involves a dynamic system of neuromuscular activity, biomechanical interactions, and sensory feedback loops that are used in synchrony to manipulate the orientation of the body's various segments and generate appropriate interaction with the environment. Postural stability is one facet of postural control that can be defined as the ability to maintain a desired orientation while resisting external and internal perturbations (Cavanaugh, Guskiewicz, $\&$ Stergiou, 2005). During quiet standing, postural sway results from a combination of inherent fluctuations in the musculoskeletal system, cardiac and respiratory variations, and neural activity. Some scholars suggest, however, that postural sway serves as an exploratory behavior for the stimulation of somatosensory and vestibular pathways to provide sensory information for increased postural control (Riley, Balasubramaniam, \& Turvey, 1999; Riley, Wong, Mitra, \& Turvey, 1997). By reconciling these two viewpoints, it is likely that sway magnitude and fluctuations are a function of the interaction between physiological states, the environment, and the implicit and explicit goals of the present task. Postural sway has been used in a number of studies as an indicator of postural stability in patients suffering from neurological deficits or disease, the elderly, and to track the development of infants (Cavanaugh, Guskiewicz, \& Stergiou, 2005; Fransson, Johansson, Hafström, \& Magnusson, 2000; Romero \& Stelmach, 2003; Van Deursen \& Simoneau, 1999; Yokoyama, Araki, Nishikitani, \& Sato, 2002). Using force plates to measure the interaction forces applied by an individual between his or her body and the plates, the individual's center of pressure (COP) can be calculated, and is used to measure postural sway.

Muscle tendon vibration has been used to introduce postural disturbances and perturbations to reveal dynamic changes in postural control in clinical and research settings (Fransson et al., 2000; Fransson, Johansson, Tjernstrom, \& Magnusson, 2003; Van Deursen \& Simoneau, 1999). Vibration of noncontracting muscles causes muscle spindle Ia afferent discharges, in synchrony with the vibration frequency, without eliciting reflex or voluntary muscle contractions (Burke, Hagbarth, Lofstedt, \& Wallin, 1976; Ribot-Ciscar, Rossi-Durand, $\&$ Roll, 1998). It was found that bilateral vibration of the Achilles tendons or calf muscles results in significant backward tilt and postural readjustment of individuals standing with an upright posture, as well as increased postural sway, as measured by deviation in COP (Barbieri et al., 2008; Ceyte et al., 2007; Hayashi, Miyake, Jijiwa, \& Watanabe, 1981; Polónyóva \& Hlavacka, 2001; Thompson, Bélanger, \& Fung, 2007). These findings indicate that muscular proprioceptive receptors contribute to the perception of body orientation and postural vertical, and that these vibratory-induced changes in peripheral somatosensory input to the central nervous system may be the main contributor to motor and perceptual disturbances (Roll, Vedel, \& Ribot, 1989).

Computerized dynamic posturography is a common tool that consists of a series of balance-control tests that investigate individual sensory and motor components of postural control and balance. The Sensory Organization Test (SOT) is a core component of the NeuroCom International (Clackamas, OR) dynamic posturography system that applies a variety of sensory conditions on upright standing posture to provide information about the integration of visual, proprioceptive, and vestibular input in postural control. One of its key postural sway measures, the Equilibrium Score (ES), is used to evaluate postural stability. This linear measure compares a participant's peak anterior-posterior center of gravity (COG-AP) sway with a theoretical limit of maximum sway attainable without falling (NeuroCom, 1991). The COG-AP used in the calculation of ES is estimated by the NeuroCom system using user-inputted participant height and weight data, and NeuroCom measured center of pressure values obtained from the force plates (see Benda, Riley, \&

Correspondence address: Charles S. Layner, University of Houston, 4800 Calhoun Rd., Houston, TX 77004, USA. e-mail address: clayne2@uh.edu 
Krebs, 1994). In addition, there are numerous linear measures of COP variability, such as displacement path length, mean and maximal range, standard deviation, and velocity, whose usefulness have been critically analyzed and demonstrated to be clinically useful (Kerr \& Eng, 2002; Raymakers, Samson, \& Verhaar, 2005; Romero \& Stelmach, 2003; Speers, Paloski, \& Kuo, 1998).

Variability in human movement is often thought of as the product of random error, and that it is minimized in healthy systems and in skilled movement. There is a growing body of support, however, for the view that variability is "not as error but as a necessary condition for function" (Harbourne $\&$ Stergiou, 2009, 269), in which variability allows for rapid adaptation to changing environments. A goal in understanding human movement is to recognize the optimal balance between too little variability, which leads to a rigid and inflexible motor control system, and too much variability, which leads to an unstable system (Stergiou, Harbourne, \& Cavanaugh, 2006). Traditional linear measures, however, are unable to represent the time-evolving structure of COP variability, especially considering recent findings suggesting that COP variation is not simply random around a central equilibrium point (Collins \& De Luca, 1993; Gagey, Martinerie, Pezard, \& Benaim, 1998; Newell, Slobounov, Slobounova, \& Molenaar, 1997). Nonlinear measures allow researchers to quantify the structure of a time series by determining the order, or predictability, of its values, with disorderly series being more complex (Cavanaugh, Guskiewicz, \& Stergiou, 2005; Harbourne \& Stergiou; Sabatini, 2000; Soames \& Atha 1982; Stergiou, Buzzi, Kurz, \& Heidel, 2004). One nonlinear analysis technique that has been applied to investigate COP variation is the calculation of approximate entropy (ApEn), a measure of time-series regularity and repeatability. By quantifying the repeatability of short sets of data within the time series as a logarithmic function, a value between 0 and 2 is generated, with lower values indicating less complexity with more repeatable and regular patterns in the time series and higher values indicating more complexity with less repeatable and regular patterns in the time series.

A number of studies have shown that extremely regular biorhythms are associated with stress and disease states (Lipsitz \& Goldberger, 1992; Vaillancourt \& Newell, 2002), and that this may reflect a reduced ability for the system in question to adapt to changes in its physiologic environment (Kaplan et al., 1991; Pikkujamsa et al., 1999; Vaillancourt \& Newell, 2000). A critical factor to support such a claim is the deterministic nature of the biorhythm being studied. A deterministic signal is one that is not simply derived from random noise, but instead stems from an ordered, nonrandom source. One perspective is that biorhythms generated by motor output are the result of complex, multicomponent interactions that occur in the nervous system (Vaillancourt \& Newell, 2003; Vaillancourt, Slifkin, \& Newell, 2001). Such a system is nonlinear because inputs induce increased variability which may elicit new motor behaviors rather than driving a linear change in motor output. Therefore, changes in the co- ordination of motor output governed by the postural-control system may be represented by changes in the complexity of postural sway.

Previous studies have shown changes in ApEn values to be independent of the behaviors evaluated by traditional linear measures and spectral analysis measures (see Cavanaugh, Guskiewicz, \& Stergiou, 2005; Sabatini, 2000). For example, when investigating seated sway patterns of infants in different developmental stages, a significant decrease in sway complexity was found while a linear analysis of COP path length and peak-to-peak anterior-posterior (AP) and medial-lateral (ML) sway amplitude was found not to be significantly different (Harbourne \& Stergiou, 2003). The authors suggest that this discrepancy reflects the adoption of an underlying stable postural strategy, by infants, through a progressive reduction in the degrees of freedom.

However, the clinical use of ApEn as an indicator of postural stability depends strongly on understanding the relationship between stability and sway complexity. Cavanaugh, Guskiewicz, and Stergiou (2005) and Cavanaugh et al. (2006) found more regular COP oscillations after cerebral concussion in athletes despite an absence of postural instability measured by ES. ApEn also took longer to return to preconcussion baseline levels than did ES. This discrepancy may reflect changes in postural control to accommodate for apparently asymptomatic neurological injury that results in additional neurophysiologic constraints. These features emphasize the use of nonlinear measures, such as ApEn, to supplement traditional linear measures, as they may reflect different components of posture control.

Spectral analysis has also been used as an additional nonlinear measure of postural sway. Dominant sway frequencies in humans are dispersed between 0 and $2.0 \mathrm{~Hz}$ (Soames \& Atha, 1982). After accounting for anthropomorphic changes, infants at various stages of development displayed greater power in lower COP frequencies $(0.4-0.5 \mathrm{~Hz})$ with increased experience, and no significant changes in peak-to-peak sway amplitude. This suggests that postural-control changes, resulting from a refinement of sensorimotor dynamics during the development phase, may indicate increased exploratory sway behavior through more frequent changes in sway direction (Chen, Metcalfe, Chang, Jeka, \& Clark, 2008). Increases in the power spectral density (PSD) profile of higher sway frequency domains in asymptomatic workers exposed to the adverse effects of neurotoxins have also been found (Yokoyama et al., 2002). As previously described, decreased ApEn measures of postural sway were observed in developing infants and are associated with stress and disease states. This may indicate that decreases in sway complexity are correlated with increases in spectral power at higher frequencies.

Interestingly, reduced support-surface stability has been found to attenuate the effect of Achilles tendon vibration on backward body displacement, but only in the AP plane. Using a see-saw rocking platform consisting of a rounded hemispherical base, the authors showed that the magnitude of backward body displacement generated by the application 
of Achilles tendon vibration on a static surface was reduced when participants stood on an unstable support surface (Ivanenko, Levik, Talis, \& Gurfinkel, 1997; Ivanenko, Solopova, \& Levik, 1999; Ivanenko, Talis, \& Kazennikov, 2000). They argued that ankle proprioceptive input no longer provides meaningful information to the postural-control system as a result of the dynamic changes in support-surface inclination. On an unstable support, ankle angle no longer accurately reflects body position relative to the Earth's gravitational gradient, and proprioceptive input alone is no longer able to detect the dynamic changes in platform orientation. To accommodate for this, the postural-control system reweights sensory input to reduce the reliance on these muscle spindle inputs. Thus, the degraded sensory information generated by tendon vibration effectively has reduced value, and therefore the influence on the postural-control system is negated. This suggests that support-surface instability modifies the role of proprioceptive information in tasks requiring bipedal postural control. In the present study, the incorporation of ApEn can more closely investigate this hypothesis because of the relationship between complexity and the physiologic components of postural sway.

By comparing and contrasting measures of COP-AP variability under different support-surface stability conditions, with and without the application of Achilles tendon vibration, insight can be gained regarding the process by which tendon vibration interferes with normal sensory information processing. Therefore, a major goal of the present study was to extend previous studies by Ivanenko et al. (1997, 2000, 1999), by obtaining both linear and nonlinear measures of COP-AP variability, a more comprehensive measure of postural sway than just body displacement (Murray, Seireg, \& Scholz, 1967), as well as the dynamic changes in neural processes revealed by linear and nonlinear measurements. Given the previous research, we expected that the addition of vibration would (a) decrease ES, (b) decrease ApEn values (more regular sway complexity), and (c) increase mean and peak sway frequencies, but that these changes would not occur in the presence of an unstable support surface.

\section{Method}

\section{Participants}

Participants were 20 young, healthy college student volunteers between the ages of 19 and 34 ( 6 women; $M$ age $=22 \pm$ 1 years, $M$ height $=173 \pm 2 \mathrm{~cm}, M$ weight $=73.9 \pm 2.82 \mathrm{~kg}$ ). Participants were free of present or previous musculoskeletal or neurological injury or deficit that might have influenced their sensory systems or posture. This was assessed through the completion of a Physical Activity Readiness Questionnaire (PAR-Q) and a self-report. Potential participants were excluded if they checked "yes" to any of the PAR-Q questions, reported any injury or deficit, or scored outside of the normative range of the SOT condition during training. The SOT disrupts individual sensory systems while maintaining upright standing posture, and performance outside the nor- mal population range would indicate a potential preexisting balance deficit.

We obtained participant demographics, anthropometric measurements, and informed consent from all participants before initiating the experiment. The study complied with the principles laid down in the Declaration of Helsinki and was approved by the University of Houston Committees for the Protection of Human Subjects.

\section{Procedure}

Prior to data collection, SOT 2 or 5 was repeatedly administered to participants randomly assigned to one of two groups (SOT2 or SOT5), using the Balance Manager Smart EquiTest system (NeuroCom, Clackamas, OR, USA). A twogroup design was selected for two reasons. First, we were concerned that the combination of two unusual stimuli might potentially produce competing or additive effects over multiple exposures to the postural-control task, even if given in a randomized order. The control condition was isolated to eliminate as many interacting variables as possible. Second, there was also concern regarding potential fatigue and learning effects from participants completing twice the number of SOT trials.

Participants were positioned standing upright on two force plates that rotated in the AP plane with foot placement according to manufacturer's instructions. COP data was collected at $100 \mathrm{~Hz}$ using a desktop computer. During SOT performance, participants stood in a quiet room to eliminate auditory distractions, closed their eyes to eliminate visual cues, crossed their arms in front of them, and maintained an upright posture with as little movement as possible. Participants were shoeless during testing and wore either shorts or pants rolled up to a height that did not interfere with the vibrators or apply pressure to the lower limbs. Participants attended two training sessions and one test session within a seven-day period. The training sessions allowed for familiarization with the task to minimize the learning effects during the test session (Wrisley et al., 2007). During the training sessions, participants only experienced the no-vibration condition.

Each SOT followed a standard protocol of three trials lasting $20 \mathrm{~s}$ each. In SOT2 participants performed with a stable support-surface platform, whereas in SOT5 the supportsurface platform was sway referenced, which tilted the platform to follow the participant's AP motion to reduce somatosensory input from the ankle musculature (NeuroCom, 1991). SOT trials were separated by a 5-s rest period. An auditory signal indicated the start of a trial after obtaining verbal readiness from the participant. Postural responses to Achilles tendon vibration have been shown not to be susceptible to expectation (Caudron, Boy, Forestier, \& Guerraz, 2008).

All participants completed their randomly assigned SOT under two conditions: (a) without vibration (NV) or (b) with vibration (V). The baseline without vibration was always presented first after which the participant was tested using 
vibration. During the training sessions, only one NV-SOT was administered, whereas both NV- and V-SOTs were administered during the test session. The test session SOTs were separated by $5 \mathrm{~min}$ of rest to control for fatigue effects. Achilles tendon vibration at $70 \mathrm{~Hz}$ was applied using one muscle tendon vibrator VB115 (TechnoConcept, Cereste, France) attached to each ankle above each Achilles tendon beginning $5 \mathrm{~s}$ prior to initiating each trial of the $\mathrm{V}$ SOT to negate the startle effect of vibration, and continuing throughout the trial's 20-s duration. Vibrators, however, were also attached throughout the NV trials to eliminate tactile discrepancies between NV- and V-SOTs.

\section{Data Reduction}

An ES for each SOT trial was generated by the EquiTest 8.0 software using COG measures calculated from COP values, shear forces, and participant height. The score was calculated as the ratio (\%) of the peak-to-peak COG-AP angular displacement to the theoretical maximum COG-AP angular displacement that an individual can sway without falling $\left(12.5^{\circ}\right)$. A value of 100 indicates perfect stability, and a value of 0 indicates a fall (NeuroCom, 1991).

Complexity of COP-AP sway was quantified by calculating ApEn values using a customized script in Matlab R2008a (Mathworks, Natick, MA). The calculation of ApEn was achieved through a moving-window technique by comparing consecutive sequences of time-series data of a given length $(m)$. The difference between a single reference sequence and all other sequences within a defined error tolerance $(r)$ gave a measure of regularity used in the calculation of ApEn. Thus, ApEn represented the likelihood that the reference sequence of data would repeat itself in the next comparison.

ApEn output values lie between 0 and 2, with higher values indicating random, complex time series (i.e., white noise) and lower values indicating repeatable, regular time series (i.e., a sine wave; Pincus, Gladstone, \& Ehrenkranz, 1991). It is important to note that there is no useful method for interpreting ApEn values measured on a single trial. During analysis, ApEn is best used as a comparative measure of change in complexity, rather than attempting to interpret its absolute value (Cavanaugh, Guskiewicz, \& Stergiou, 2005).

The following parameters were selected and used in the determination of ApEn values for COP data in the AP plane: (a) a series length of $2(m=2)$, (b) error tolerance of 0.2 times the series' standard deviation $(r=0.2 \times \sigma)$, and (c) a lag value of 10 . Given the selected parameters and a data collection rate of $100 \mathrm{~Hz}$, previous research and guidelines have shown that the number of COP-AP data points obtained ( $n=2,000)$ was sufficient to differentiate between data sets based on regularity (Cavanaugh, Guskiewicz, Giuliani, et al., 2005, 2006; Pincus, Gladstone, \& Ehrenkranz, 1991; Stergiou et al., 2004).

Nonlinearity of COP-AP data needs to be established to justify the use of a nonlinear measure such as ApEn. Surrogate data is a well-established means to test for nonlinearity as it possesses the same characteristics (variance, mean, and power spectra) as the COP-AP data but is randomly generated (Schreiber \& Schmitz, 2000). Associated surrogate data for each participant's COP-AP data were generated in Matlab R2008a (Mathworks, Natick, MA) using the Iterative Amplitude Adaptive Fourier Transform algorithm described in Schreiber and Schmitz's (1996) study. Two-tailed rank testing of a nonlinearity test statistic computed for each participant's COP-AP data against surrogate data revealed a significant difference in means for all SOT trials in both NV and V conditions ( $p=.039$; Theiler, Eubank, Longtin, Galdrikian, \& Doyne Farmer, 1992). This indicates that none of the COP-AP data were derived from a random source and that ApEn is a valid nonlinear measure.

Mean and peak frequency values were obtained from each participant's COP-AP PSD to quantify the change in sway direction under different sensory conditions. An average PSD was computed from the three SOT trials to reveal the strength of AP sway frequencies between 0 and $5.0 \mathrm{~Hz}$. The mean frequency value of a given participant's COP-AP data was the frequency generating the average power determined from the PSD, whereas the peak frequency value was the frequency that displays the greatest power in the PSD. Both values were obtained for comprehensiveness as they are mutually exclusive of each other, and change in one may not necessarily accompany a change in the other. COP-AP frequency analysis was conducted using Sigview 1.98 (SignalLab, Pforzheim, Germany). Previous research has indicated that $99 \%$ of COPAP frequency power lies well below $5.0 \mathrm{~Hz}$ (Chen et al., 2008; Mezzarane \& Kohn, 2008; Soames \& Atha, 1982; Yokoyama et al., 2002). This is consistent with $98 \%$ of the power lying below $5.0 \mathrm{~Hz}$ in our data set. Given that the dominant sway frequencies in humans lie between 0.0 and $2.0 \mathrm{~Hz}$ (Soames \& Atha), a conservative second-order Butterworth low-pass filter at $5.0 \mathrm{~Hz}$ was applied to the COP-AP data after smoothing using a Hann data window of 15 points. This was to remove high-frequency noise present in the digitized kinematic data while maintaining the integrity of the data generated by the postural sway.

\section{Data Analysis}

ES and ApEn were averaged across each participant's SOT trials for each vibration condition. Mean and peak frequency values were already averaged in the computation of each participant's PSD.

A mixed two-way analysis of variance (ANOVA) was conducted on all four dependent variables (ES, ApEn, mean frequency, and peak frequency), with the null hypotheses that there were no changes in their mean values $(p=.05)$. The within-participants factor was the vibration condition (NV and $\mathrm{V}$ ) and the between-participants factor was the support stability (SOT2 and SOT5). When an interaction was seen, a post hoc analysis was conducted using a Student's $t$ test with an adjusted alpha value of .025 to account for multiple comparisons and reduce the likelihood of making a family-wise Type I error. All statistical analyses were performed using SPSS (Version 17.0). 


\begin{tabular}{|c|c|c|c|}
\hline Group & SOT2 & SOT5 & $M$ \\
\hline \multicolumn{4}{|l|}{ ES } \\
\hline NV & $92.80 \pm 0.70$ & $66.60 \pm 3.90$ & $79.70 \pm 2.20$ \\
\hline $\mathrm{V}$ & $84.60 \pm 2.10$ & $64.70 \pm 4.00$ & $74.60 \pm 2.90$ \\
\hline$M$ & $88.70 \pm 1.40$ & $65.70 \pm 3.60$ & - \\
\hline \multicolumn{4}{|l|}{ ApEn } \\
\hline $\mathrm{NV}$ & $0.60 \pm 0.05$ & $0.48 \pm 0.04$ & $0.54 \pm 0.04$ \\
\hline $\mathrm{V}$ & $0.41 \pm 0.05$ & $0.47 \pm 0.04$ & $0.44 \pm 0.04$ \\
\hline$M$ & $0.50 \pm 0.05$ & $0.48 \pm 0.04$ & - \\
\hline \multicolumn{4}{|c|}{ Mean frequency $(\mathrm{Hz})$} \\
\hline NV & $0.92 \pm 0.03$ & $0.85 \pm 0.07$ & $0.89 \pm 0.05$ \\
\hline V & $1.03 \pm 0.04$ & $1.11 \pm 0.10$ & $1.07 \pm 0.06$ \\
\hline$M$ & $0.98 \pm 0.03$ & $0.98 \pm 0.08$ & - \\
\hline \multicolumn{4}{|c|}{ Peak frequency $(\mathrm{Hz})$} \\
\hline NV & $0.22 \pm 0.03$ & $0.17 \pm 0.04$ & $0.19 \pm 0.03$ \\
\hline $\mathrm{V}$ & $0.38 \pm 0.04$ & $0.40 \pm 0.17$ & $0.39 \pm 0.10$ \\
\hline$M$ & $0.30 \pm 0.04$ & $0.28 \pm 0.09$ & - \\
\hline
\end{tabular}

Note. $\mathrm{ES}=$ equilibrium scores; $\mathrm{NV}=$ without vibration; $\mathrm{V}=$ vibration; $\mathrm{ApEn}=$ approximate entropy.

\section{Results}

All participants completed the two training sessions and test session without scoring within the clinical range on any of the trials, and no participant required external intervention to prevent falling while completing a SOT. In addition, Student's $t$ tests ( $p=.025$ ) conducted on mean ES, ApEn, and mean and peak frequency values on the first and last SOT test trial, for each condition ( $\mathrm{NV}$ and $\mathrm{V}$ ), revealed no significant differences, suggesting that no learning effect was present. Mean ES, ApEn, and mean and peak frequency values for each group and condition are reported in Table 1.

\section{Equilibrium Score}

The data presented in Figure 1 indicate that the application of vibration, regardless of the support surface, resulted in an increase in postural sway as reflected in decreased ES score, $F(1,18)=10.152, p=.005$. However, this effect did not depend on support-surface stability as was hypothesized, as no significant interaction was found between group and condition, $F(1,18)=3.882, p=.064$. Participants also displayed much greater postural sway when attempting to maintain posture on an unstable support surface in comparison with a stable surface (SOT5 vs. SOT2) regardless of whether vibration was applied, $F(1,18)=39.875, p<.001$.

\section{COP-AP Sway Complexity}

Consistent with the above findings, sway complexity was also reduced with the addition of vibration, $F(1,18)=$ $14.899, p=.001$, but no main effect of support stability on
ApEn was observed. This stands in contrast to the ES scores that indicated COP-AP sway magnitude was much greater in the unstable support-surface group (i.e., SOT5) relative to the stable support-surface group (i.e., SOT2). There was, however, an interaction between vibration and group that is

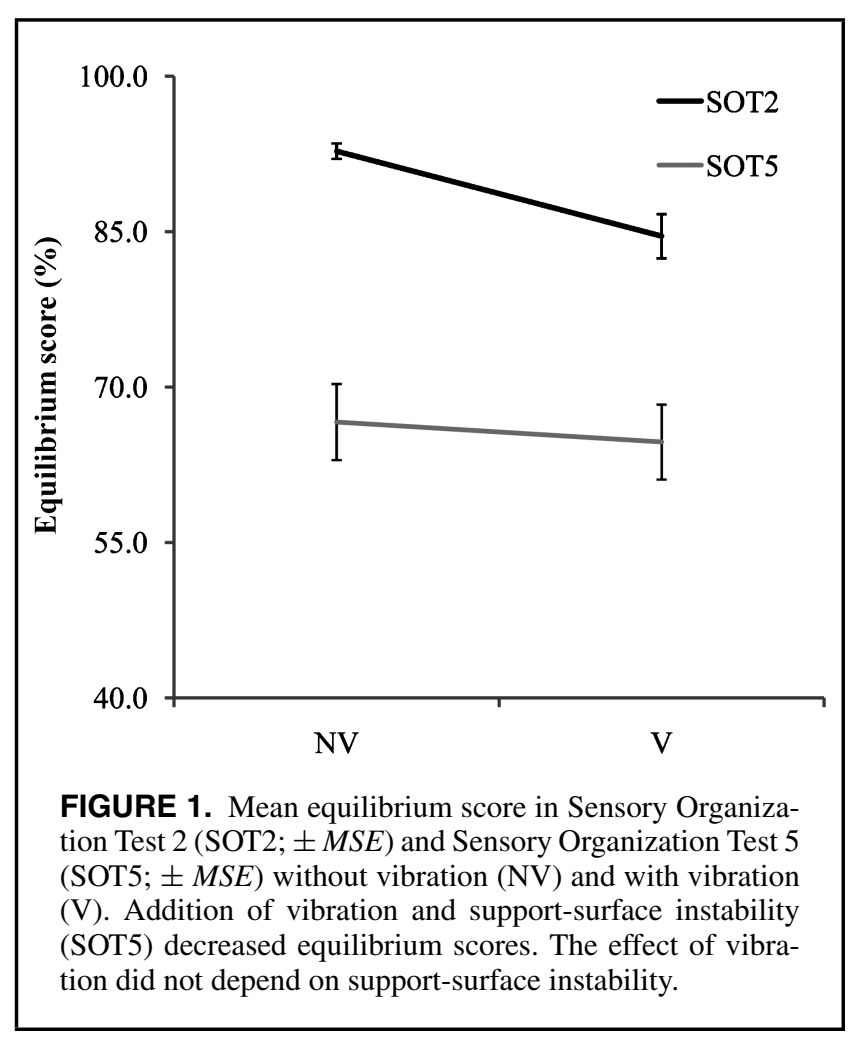


reflected in Figure 2, $F(1,18)=13.427, p=.002$. Consistent with the hypothesis, results from a post hoc analysis indicate that the addition of vibration had no impact on complexity of COP-AP sway when on an unstable support surface but complexity was decreased when the task was performed on a stable support surface, $t(10)=2.6836, p=.015$. When vibration was applied sway complexity in SOT2 was reduced, such that it approximated the complexity observed in SOT5.

\section{Frequency Analysis}

The results of the frequency analysis mirror those obtained from the ApEn data. The results of the ANOVA revealed a main effect of vibration on mean and peak frequency values, although there was no significant main effect of group: mean frequency, $F(1,18)=29.430, p<.001$; peak frequency: $F(1$, $18)=7.938, p=.011$. This suggests that the frequency of AP postural sway is independent of support-surface stability (Figure 3), but increases with the addition of vibration. At variance with the hypothesis, an interaction was found between group and condition for mean COP-AP frequencies, $F(1,18)=4.603, p=.046$, indicating that the effect of vibration is greater on an unstable support surface. The data presented in Figure 4 suggest that spectral power for both groups increased across frequencies with the addition of vibration with the greatest change at the higher frequencies.

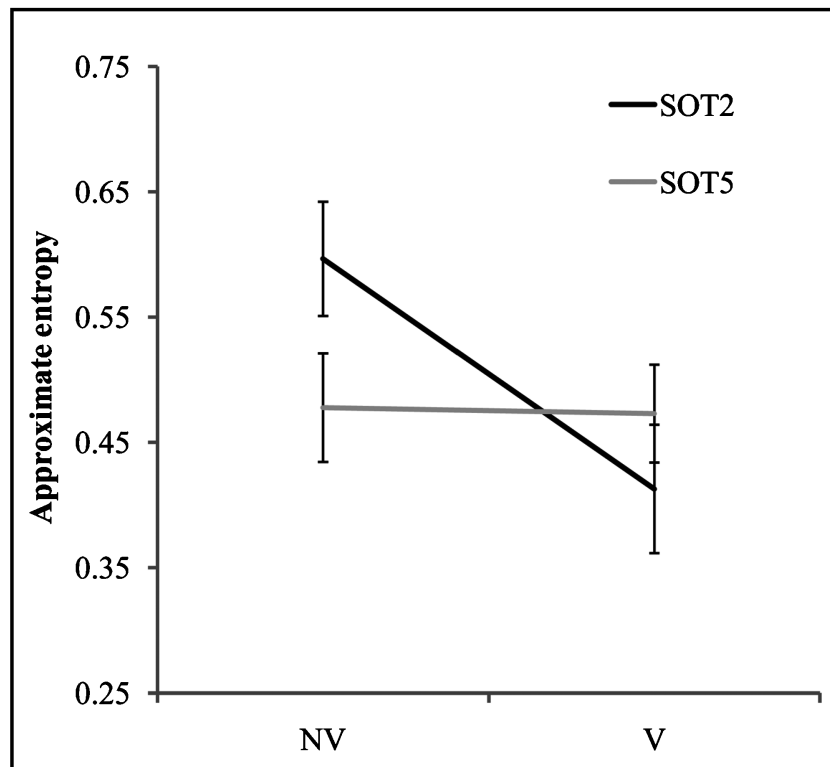

FIGURE 2. Approximate entropy scores in Sensory Organization Test 2 (SOT2; $\pm M S E$ ) and Sensory Organization Test 5 (SOT5; \pm MSE) without vibration (NV) and with vibration $(\mathrm{V})$. The addition of vibration decreased complexity, but only in the stable-support condition (SOT2).

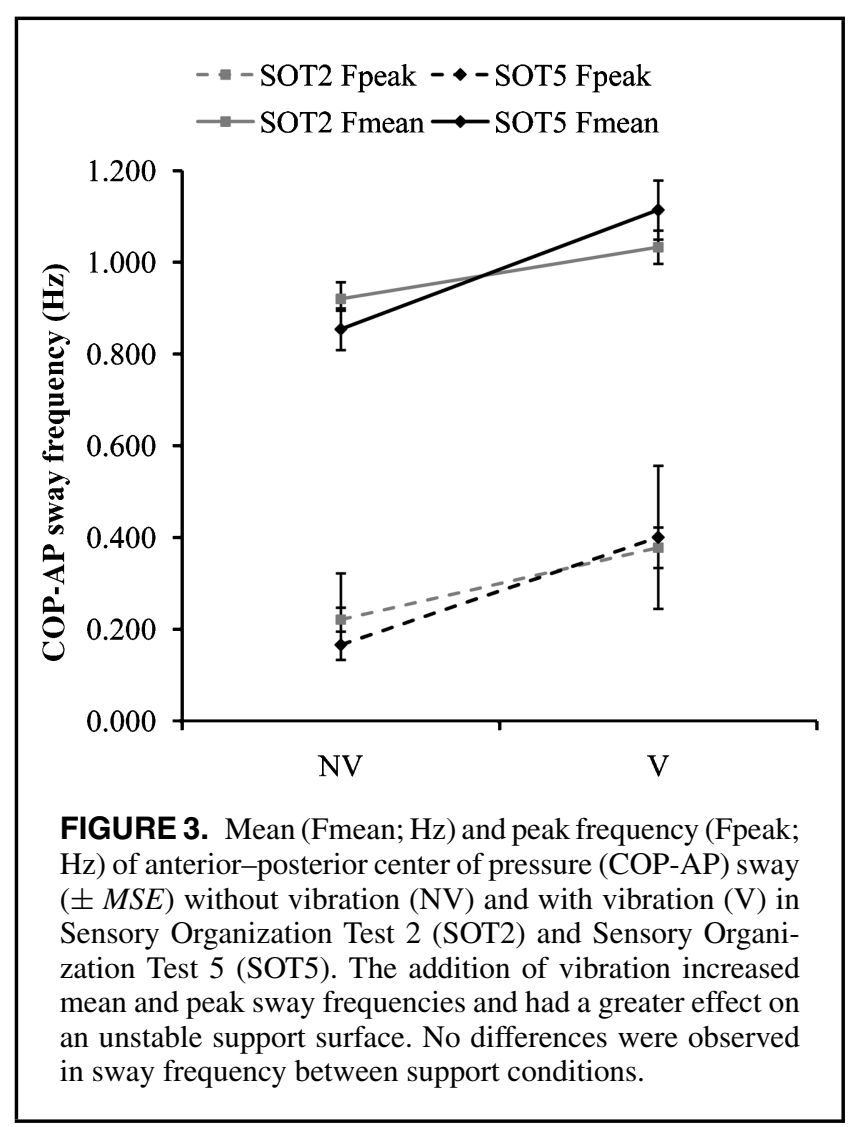

\section{Discussion}

In the present study, we investigated the role of ankle muscular spindle input on postural control by the use of Achilles tendon vibration during a bipedal postural-control task on either a stable or unstable support surface. Additionally, we evaluated the possibility of linear and nonlinear measures of COP-AP to determine if the two type of measures provided potentially different information about postural-control characteristics. In the present study, SOT 5 removed useful ankle musculature spindle input using a sway-referenced platform similar to the rocking platform used by Ivanenko et al. (1999). With an unstable support, muscle spindle input s insufficient to detect changes in platform inclination, which is required to maintain the body's COG over the absolute vertical. In fact, while on a movable support surface, ankle angle no longer corresponds to a meaningful body orientation relative to the absolute vertical. Ivanenko et al. argued that sensory reweighting occurs to reduce the reliance on muscle spindle inputs from the ankle in the presence of an unstable support surface, thus reducing the effect of degraded sensory input generated by tendon vibration. The suggestion of sensory reweighting might offer an explanation why ES and ApEn scores were unaffected in SOT5 between the baseline and vibration measures. Given the possibility that the reliance on ankle musculature spindle input is already minimized in an unstable support-surface condition, the application of Achilles tendon vibration would not have the same impact as 


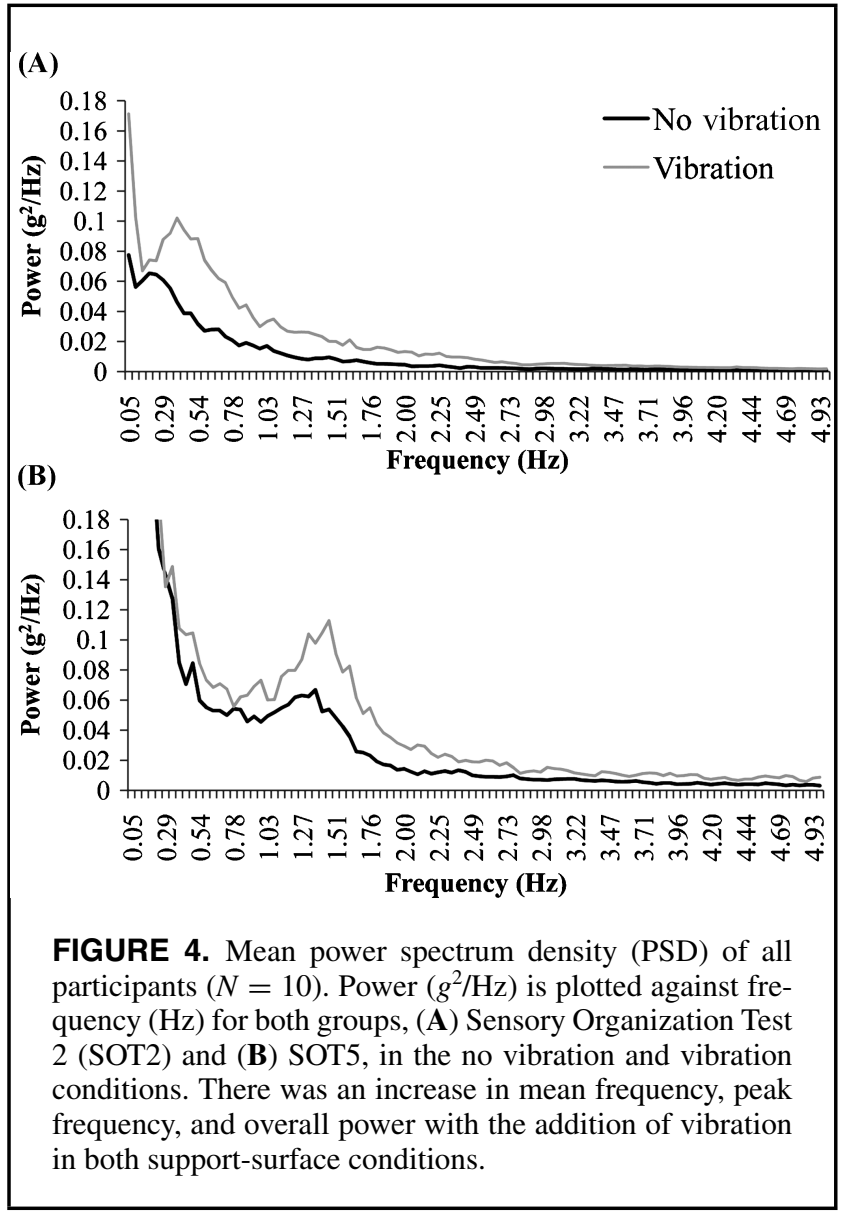

that observed in a stable support-surface condition in which spindle input would be of greater importance to postural control. It may be of interest to determine if the postural-control system could reweight ankle musculature spindle input during vibration on a stable support surface, such that with repeated exposures to vibration sway complexity would not decrease, as was found in the present investigation.

The present study extends previous research into the effect of Achilles tendon vibration and support-surface characteristics on postural stability by introducing nonlinear measures of postural sway complexity (ApEn) and sway frequency. It has been suggested that nonlinear measures of postural sway may better reflect changes in the complex, multicomponent interactions that take place in the postural-control system due to the inability of a linear measure such as ES to capture the dynamic temporal changes of postural sway (Cavanaugh, Guskiewicz, Giuliani, et al., 2005; Chaudhry et al., 2004; Sabatini, 2000; Soames \& Atha, 1982). The analysis revealed four main findings: (a) the effect of vibration on the magnitude of postural sway did not depend on support-surface stability, (b) the effect of vibration on sway complexity was only present under stable support conditions, (c) there was no difference in sway complexity between stable and unstable support conditions with tendon vibration, and (d) the addition of vibration increased mean and peak sway frequency, but had a greater effect on an unstable support surface.

There were several limitations to this study, beyond the small sample size. First, all of the study participants were fairly young and college aged. Some studies have shown changes in motor output complexity related to aging and the elderly (Lipsitz \& Goldberger, 1992), as well as throughout infant development (Harbourne \& Stergiou, 2003). Future researchers should investigate similar phenomena among people of varied ages. We also divided the participants into two groups, with each participant performing an SOT with and without vibration. To enhance internal validity and reduce between-participant variability each participant should only be assigned one of the four possible combinations of support stability and vibration. Finally, participants who performed on an unstable support surface were exposed to ankle vibration immediately prior to initiation of the SOT. This might have allowed them some small window of opportunity of adaptation for their postural-control system on a stable support surface. Initiating tendon vibration after the support surface becomes unstable may have some detectable effect on sway complexity.

We found that the addition of vibration decreased ES in the stable (SOT2) and unstable support-surface conditions (SOT5). However, finding no significant interaction between support surface and vibration suggests that the effect of vibration does not depend on support stability. This contradicts previous reports that backward body displacement resulting from vibration was mitigated by support-surface instability (Ivanenko et al., 1997, 2000, 1999). Trends in the data indicate this may still be the case, but a small sample size may have contributed to a Type II error: failure to reject the null hypothesis when the null hypothesis is false.

On the other hand, results from the analysis of ApEn were exactly as predicted. The effect of vibration on sway complexity was dependent on support-surface stability and could only be observed under stable support-surface conditions, as we had also expected to occur with ES. More interestingly, however, is that no differences in sway complexity, mean frequency, or peak frequency were found between stable and unstable support-surface conditions with vibration. This effect may suggest that an increase in sway amplitude, measured by ES, does not necessarily correspond to a decrease in postural stability, and that sway complexity represented by nonlinear measures of sway may be a more complete indicator of postural stability. If this is the case, these results suggest that despite Achilles tendon vibration, postural stability on an unstable support surface is at least as effective as on a stable support surface. One possible explanation is that even with the addition of vibration, a shift in the weighting of sensory input in the unstable support-surface condition to accommodate for reduced ankle proprioceptive input results in an insignificant change in the overall system dynamics underlying postural control. Furthermore, this finding supports previous research into the clinical utility of ApEn to assess the recovery of asymptomatic athletes who have sustained a 
cerebral concussion but display no changes in traditional measures of postural stability (Cavanaugh, Guskiewicz, \& Stergiou, 2005; Cavanaugh, Guskiewicz, Giuliani, et al., 2005; Cavanaugh et al., 2006).

In conclusion, results from the present study showed that linear and nonlinear measures of postural sway may reveal contrary or varied results. Nonlinear measures may express fundamental dynamic components of the posturalcontrol system that are not captured by traditional linear measures. This property of nonlinear measures suggests that they could be better indicators of postural stability. Future researchers should strongly consider multiple measures of postural sway (linear and nonlinear) when assessing changes in the postural-control system. A broader knowledge base in this regard would clarify the specific characteristics of the relationship between nonlinear measures of postural sway and the postural-control system.

\section{ACKNOWLEDGMENTS}

The authors wish to thank Sheryl Bishop for her statistical support in this project and Amir Pourmoghaddam for his development of customized Matlab scripts.

\section{REFERENCES}

Barbieri, G., Gissot, A. S., Fouque, F., Casillas, J. M., Pozzo, T., \& Pérennou, D. (2008). Does proprioception contribute to the sense of verticality? Experimental Brain Research, 185, 545-552.

Benda, B. J., Riley, P. O., \& Krebs, D. E. (1994). Biomechanical relationship between center of gravity and center of pressure during standing. IEEE Transactions on Rehabilitation Engineering, 2, 3-10.

Burke, D., Hagbarth, K. E., Lofstedt, L., \& Wallin, B. G. (1976). The responses of human muscle spindle endings to vibration of noncontracting muscles. The Journal of Physiology, 261, 673-693.

Caudron, S., Boy, F., Forestier, N., \& Guerraz, M. (2008). Influence of expectation on postural disturbance evoked by proprioceptive stimulation. Experimental Brain Research, 184, 53-59.

Cavanaugh, J. T., Guskiewicz, K. M., \& Stergiou, N. (2005). A nonlinear dynamic approach for evaluating postural control: New directions for the management of sport-related cerebral concussion. Sports Medicine, 35, 935-950.

Cavanaugh, J. T., Guskiewicz, K. M., Giuliani, C., Marshall, S., Mercer, V., \& Stergiou, N. (2005). Detecting altered postural control after cerebral concussion in athletes with normal postural stability. British Journal of Sports Medicine, 39, 805-811.

Cavanaugh, J. T., Guskiewicz, K. M., Giuliani, C., Marshall, S., Mercer, V. S., \& Stergiou, N. (2006). Recovery of postural control after cerebral concussion: New insights using approximate entropy. Journal of Athletic Training, 41, 305-313.

Ceyte, H., Cian, C., Zory, R., Barraud, P. A., Roux, A., \& Guerraz, M. (2007). Effect of Achilles tendon vibration on postural orientation. Neuroscience Letters, 416(1), 71-75.

Chaudhry, H., Findley, T., Quigley, K. S., Bukiet, B., Ji, Z., Sims, T., et al. (2004). Measures of postural stability. Journal of Rehabilitation Research and Development, 41, 713-720.

Chen, L. C., Metcalfe, J. S., Chang, T. Y., Jeka, J. J., \& Clark, J. E. (2008). The development of infant upright posture: sway less or sway differently? Experimental Brain Research, 186, 293303.
Collins, J. J., \& Luca, C. J. (1993). Open-loop and closed-loop control of posture: A random-walk analysis of center-of-pressure trajectories. Experimental Brain Research, 95(2), 308-318.

Fransson, P. A., Johansson, R., Hafström, A., \& Magnusson, M. (2000). Methods for evaluation of postural control adaptation. Gait \& Posture, 12(1), 14-24.

Fransson, P. A., Tjernström, F., Hafström, A., Magnusson, M., \& Johansson, R. (2002). Analysis of short-and long-term effects of adaptation in human postural control. Biological Cybernetics, 86, $355-365$.

Fransson, P. A., Johansson, R., Tjernstrom, F., \& Magnusson, M. (2003). Adaptation to vibratory perturbations in postural control. Engineering in Medicine and Biology, IEEE, 22, 53-57.

Gagey, P. M., Martinerie, J., Pezard, L., \& Benaim, C. (1998). Static balance is controlled by a non-linear dynamic system. Annales D'oto-Laryngologie Et De Chirurgie Cervico Faciale: Bulletin De La Société D'oto-Laryngologie Des Hôpitaux De Paris [Annals of Otolaryngology and Cervico-Facial Surgery: Bulletin of the Paris Hospitals Society of Otolarynology], 115, 161-168.

Harbourne, R. T., \& Stergiou, N. (2003). Nonlinear analysis of the development of sitting postural control. Developmental Psychobiology, 42, 368-377.

Harbourne, R. T., \& Stergiou, N. (2009). Movement variability and the use of nonlinear tools. Physical Therapy, 89, 267-282.

Hayashi, R., Miyake, A., Jijiwa, H., \& Watanabe, S. (1981). Postural readjustment to body sway induced by vibration in man. Experimental Brain Research, 43, 217-225.

Ivanenko, Y. P., Levik, Y. S., Talis, V. L., \& Gurfinkel, V. S. (1997). Human equilibrium on unstable support: the importance of feetsupport interaction. Neuroscience Letters, 235, 109-112.

Ivanenko, Y. P., Solopova, I. A., \& Levik, Y. S. (2000). The direction of postural instability affects postural reactions to ankle muscle vibration in humans. Neuroscience Letters, 292, 103-106.

Ivanenko, Y. P., Talis, V. L., \& Kazennikov, O. V. (1999). Support stability influences postural responses to muscle vibration in humans. European Journal of Neuroscience, 11, 647-654.

Kaplan, D. T., Furman, M. I., Pincus, S. M., Ryan, S. M., Lipsitz, L. A., \& Goldberger, A. L. (1991). Aging and the complexity of cardiovascular dynamics. Biophysical Journal, 59, 945-949.

Kerr, H. M., \& Eng, J. J. (2002). Multidirectional measures of seated postural stability. Clinical Biomechanics, 17(7), 555-557.

Lipsitz, L. A., \& Goldberger, A. L. (1992). Loss of "complexity" and aging. Journal of the American Medical Association, 287, 1805-1809.

Mezzarane, R. A., \& Kohn, A. F. (2008). Postural control during kneeling. Experimental Brain Research, 187, 395-405.

Murray, M. P., Seireg, A., \& Scholz, R. C. (1967). Center of gravity, center of pressure, and supportive forces during human activities. Journal of Applied Physiology, 23, 831-838.

NeuroCom. (1991). Equitest System Data Interpretation Manual. Clackmas, OR: NeuroCom International.

Newell, K. M., Slobounov, S. M., Slobounova, E. S., \& Molenaar, P. C. (1997). Stochastic processes in postural center-of-pressure profiles. Experimental Brain Research, 113, 158-164.

Pikkujamsa, S. M., Makikallio, T. H., Sourander, L. B., Raiha, I. J., Puukka, P., Skytta, J., et al. (1999). Cardiac interbeat interval dynamics from childhood to senescence: Comparison of conventional and new measures based on fractals and chaos theory. Circulation, 100, 393-399.

Pincus, S. M., Gladstone, I. M., \& Ehrenkranz, R. A. (1991). A regularity statistic for medical data analysis. Journal of Clinical Monitoring and Computing, 7, 335-345.

Pincus, S. M., \& Goldberger, A. L. (1994). Physiological timeseries analysis: What does regularity quantify? American Journal of Physiology-Heart and Circulatory Physiology, 266, 1643-1656. 
Polónyová, A., \& Hlavacka, F. (2001). Human postural responses to different frequency vibrations of lower leg muscles. Physiology Research, 50, 405-410.

Raymakers, J. A., Samson, M. M., \& Verhaar, H. J. (2005). The assessment of body sway and the choice of the stability parameter(s). Gait \& Posture, 21, 48-58.

Ribot-Ciscar, E., Rossi-Durand, C., \& Roll, J. P. (1998). Muscle spindle activity following muscle tendon vibration in man. $\mathrm{Neu}$ roscience Letters, 258, 147-150.

Riley, M. A., Balasubramaniam, R., \& Turvey, M. T. (1999). Recurrence quantification analysis of postural fluctuations. Gait \& Posture, 9, 65-78.

Riley, M. A., Wong, S., Mitra, S., \& Turvey, M. T. (1997). Common effects of touch and vision on postural parameters. Experimental Brain Research, 117, 165-170.

Roll, J. P., Vedel, J. P., \& Ribot, E. (1989). Alteration of proprioceptive messages induced by tendon vibration in man: A microneurographic study. Experimental Brain Research, 76, 213222.

Romero, D. H., \& Stelmach, G. E. (2003). Changes in postural control with aging and Parkinson's disease. Engineering in Medicine and Biology Magazine, IEEE, 22(2), 27-31.

Sabatini, A. M. (2000). Analysis of postural sway using entropy measures of signal complexity. Medical and Biological Engineering and Computing, 38, 617-624.

Schreiber, T., \& Schmitz, A. (1996). Improved surrogate data for nonlinearity tests. Physical Review Letters, 77, 635-638.

Schreiber, T., \& Schmitz, A. (2000). Surrogate time series. Physica D: Nonlinear Phenomena, 142, 346-382.

Soames, R. W., \& Atha, J. (1982). The spectral characteristics of postural sway behaviour. European Journal of Applied Physiology, 49, 169-177.

Speers, R. A., Paloski, W. H., \& Kuo, A. D. (1998). Multivariate changes in coordination of postural control following spaceflight. Journal of Biomechanics, 31, 883-889.

Stergiou, N., Buzzi, U. H., Kurz, M. J., \& Heidel, J. (2004). Nonlinear tools in human movement. In R. Stergiou (Ed.), Innovative analyses of human movement: Analytical tools for human movement research (pp. 63-90). Champaign, IL: Human Kinetics.

Stergiou, N., Harbourne, R. T., \& Cavanaugh, J. T. (2006). Optimal movement variability: A new theoretical perspective for neuro- logic physical therapy. Journal of Neurologic Physical Therapy, 30, 120-129.

Theiler, J., Eubank, S., Longtin, A., Galdrikian, B., \& Doyne Farmer, J. (1992). Testing for nonlinearity in time series: The method of surrogate data. Physica D: Nonlinear Phenomena, 58(1-4), 77-94.

Thompson, C., Bélanger, M., \& Fung, J. (2007). Effects of bilateral Achilles tendon vibration on postural orientation and balance during standing. Clinical Neurophysiology, 118, 2456-2467.

Vaillancourt, D. E., \& Newell, K. M. (2000). The dynamics of resting postural tremor in Parkinson's disease. Clinical Neurophysiology, 111, 2046-2056.

Vaillancourt, D. E., \& Newell, K. M. (2002). Changing complexity in human behavior and physiology through aging and disease. Neurobiology of Aging, 23, 1-11.

Vaillancourt, D. E., \& Newell, K. M. (2003). Effects of aging on force variability, single motor unit discharge patterns, and the structure of 10,20, and $40 \mathrm{~Hz}$ EMG activity. Neurobiology of Aging, 24(1), 25-35.

Vaillancourt, D. E., Slifkin, A. B., \& Newell, K. M. (2001). Visual control of isometric force output in Parkinson's disease. Society for Neuroscience Abstracts, 27(2), 1410-1418.

Van Deursen, R. W., \& Simoneau, G. G. (1999). Foot and ankle sensory neuropathy, proprioception, and postural stability. The Journal of Orthopaedic and Sports Physical Therapy, 29, 718-726.

Wrisley, D. M., Stephens, M. J., Mosley, S., Wojnowski, A., Duffy, J., \& Burkard, R. (2007). Learning effects of repetitive administrations of the sensory organization test in healthy young adults. Archives of Physical Medicine and Rehabilitation, 88, 1049-1054.

Yokoyama, K., Araki, S., Nishikitani, M., \& Sato, H. (2002). Computerized posturography with sway frequency analysis: Application in occupational and environmental health. Industrial Health, 40(1), 14-22.

Submitted March 19, 2009

Revised July 23, 2009

Second revision September 25, 2009

Accepted September 30, 2009 